\title{
Les erratiques de dolomie sur le rivage des Escoumins, Côte Nord de l'estuaire maritime du Saint-Laurent, Québec
}

\author{
Jean-Claude Dionne et Pascal Bernatchez \\ Département de géographie et Centre d'études nordiques, Université Laval, \\ Québec, $Q C$ GIK $7 P 4$ \\ Reçu: le 6 décembre, 2000 \\ Accepté: le 7 mai, 2001
}

\begin{abstract}
Le rivage entre Les Escoumins et Baie-des-Bacons, sur la Côte Nord du Saint-Laurent, est caractérisé par une large batture $(800$ à $2000 \mathrm{~m})$ argileuse et une falaise entaillant la terrasse de $30 \mathrm{~m}$ constituée de dépôts meubles quaternaires. Il est parsemé de milliers de cailloux, de lithologies et de tailles variées, formant des dallages sur le bas estran et des amas divers sur le haut du rivage, au pied de la falaise.

La presque totalité des cailloux (plus de $90 \%$ ) appartient aux diverses lithologies ignées et métamorphiques du socle précambrien à proximité. Mais on trouve aussi de nombreux cailloux de dolomie, une lithologie rare dans le Bouclier Laurentidien. Sur une distance de $18 \mathrm{~km}, 3661$ cailloux de dolomie ont été observés et mesurés. Il en existe plusieurs variétés; du point de vue de la couleur, les dolomies grises et roses dominent, totalisant respectivement $74 \%$ et $20 \%$. Parmi les autres caractéristiques, il y a une trentaine de blocs de dolomie à stromatolites, plus de $28 \%$ des cailloux de dolomies observés sont striés et près de la moitié sont subarrondis. Les cailloux de petite taille $(1 \mathrm{à} 10 \mathrm{~kg})$ comptent pour environ $59 \%$, ceux de 10 à $50 \mathrm{~kg}$, pour $30 \%$, ceux de 50 à $100 \mathrm{~kg}$. $7 \%$, alors que $4 \%$ pèsent plus de $100 \mathrm{~kg}$, le plus gros atteignant deux tonnes. Les deux plus gros blocs de dolomie à stromatolites pèsent respectivement $175 \mathrm{et} 280 \mathrm{~kg}$.

Il existe quatre grandes aires de roches dolomitiques dans le Bouclier précambrien: le bassin sédimentaire de Mistassini, le secteur entre la Grande île et Inukjuak, sur la côte est de la mer d'Hudson, le fossé du Labrador, ainsi que dans le Grenville, entre Hull et le lac Bastong. La source la plus plausible des erratiques de dolomie des Escoumins demeure le bassin de Mistassini, ce qui implique un courant de glace important, au Wisconsinien, vers le SE à partir d'un dôme ou d'une ligne de partage des glaces situé au NO du lac Mistassini. Les erratiques de dolomie du rivage des Escoumins ont d'abord été transportés par le glacier via la dépression du Lac-Saint-Jean et du Saguenay avant d'être relâchés par les icebergs ou peut-être même, en partie, par une plate-forme de glace localisée en bordure de la Mer de Goldthivait. Les déplacements sont de l'ordre de 400 à $500 \mathrm{~km}$. Par ailleurs, plusieurs blocs ont pu être déplacés localement par les glaces flottantes au cours de l'Holocène.
\end{abstract}

The shoreline between Les Escoumins and Baie-des-Bacons, on the North Shore of the Lower St. Lawrence. is characterized by a wide clayey tidal flat $(800$ to $2000 \mathrm{~m})$ and a cliff cut into the $30-\mathrm{m}$ terrace made of Quaternary unconsolidated deposits. The shore zone is covered by thousands of boulders of various lithologies and sizes, including mega-boulders scattered throughout the tidal flat, small boulders $(25$ to $40 \mathrm{~cm})$ commonly forming pavements at the surface of the tidal flat, and boulders of various sizes on the beach at the base of the cliff.

Most erratics are Precambrian igneous and metamorphic rocks from the adjacent Shield area; however, dolostone erratics are also a significant component. In an area $18 \mathrm{~km}$ long, 3661 dolostone erratics were observed and measured. There are various types of dolostone including 30 stromatolitic dolostone erratics; most are grey (74\%) and pink $(20 \%)$. Among other characteristics, $28 \%$ of the erratics are striated and about half are subrounded; small size erratics ( 1 to $10 \mathrm{~kg}$ ) account for about $59 \%$, those 10 to $50 \mathrm{~kg}$, for $30 \%$, those 50 to $100 \mathrm{~kg}, 7 \%$, while $4 \%$ weigh over $100 \mathrm{~kg}$, the largest boulder weighing two tons. The two largest stromatolitic dolostone erratics weigh respectively 280 and $175 \mathrm{~kg}$.

There are four areas in the Precambrian Shield were dolostone formations occur: the sedimentary basin of Mistassini, the east coast of Hudson Bay, between Long Island and Inukjuak, the Labrador Trough, and a wide area in the Grenville between Hull and Lake Bastong. The most likely source of the dolostone erratics observed at Les Escoumins is the Mistassini sedimentary basin. This interpretation implies a major ice flow to the SE during the Wisconsinan from an ice dome or an ice devide located to the NW of Lake Mistassini. The erratics occurring in the area of Les Escoumins were likely first transported by a glacier via the Lake St. Jean-Saguenay corridor before being released in the Goldthwait Sea most probably by icebergs or possibly by a nearby ice shelf. Distances of displacement range from 400 to $500 \mathrm{~km}$. Many erratics may also have been moved locally by shore ice during the Holocene.

\section{INTRODUCTION}

En raison des événements géologiques survenus au Quaternaire, en particulier au Wisconsinien et à l'Holocène, les rivages du Saint-Laurent estuarien sont, à peu près partout, couverts de cailloux de lithologies très variées (Dionne 1972, 1979, 1987, 1991). Certaines lithologies relativement rares et géographiquement peu répandues peuvent donc servir 


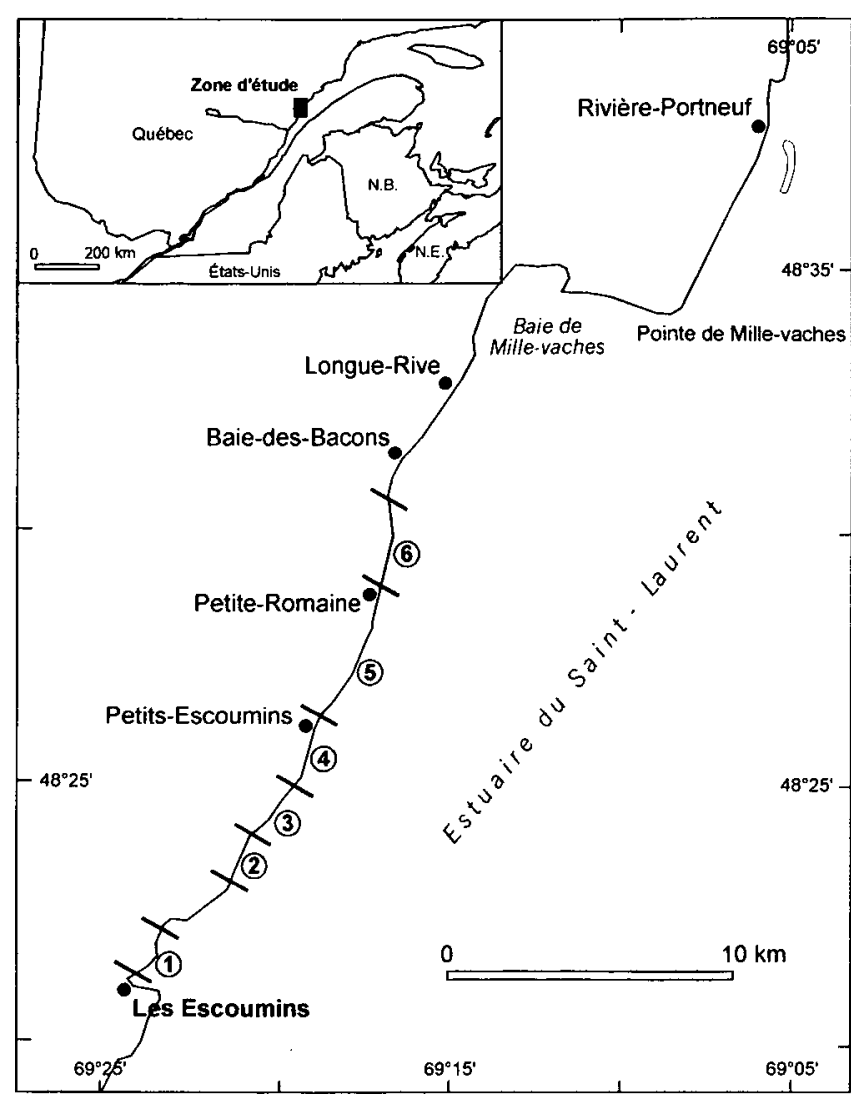

Fig. 1. Carte de localisation du site étudié et des secteurs inventoriés.

d'indicateurs d'un transport par les glaciers, les icebergs ou encore les glaces flottantes.

Sur les rivages voisins de l'embouchure du Saguenay, par exemple, on trouve des cailloux de dolomie à stromatolites (Dionne 1986, 1994) qui proviennent du bassin sédimentaire de Mistassini, d'âge protérozoïque. Les étendues connues de roches dolomitiques avec ou sans stromatolites étant peu nombreuses dans le Bouclier Laurentidien (Avramtchev 1985), cette lithologie constitue alors un indicateur précieux de l'écoulement des glaces (Veillette 1999).

Sur la rive nord de l'estuaire, l'aire de distribution des erratiques de dolomie n'est toutefois pas restreinte à l'embouchure du Saguenay. On en trouve aussi dans d'autres secteurs, notamment celui des Escoumins situé à plus de 40 $\mathrm{km}$ au NE de Tadoussac, ainsi qu'à Cap-Colombier, à plus de $140 \mathrm{~km}$. Cette dernière localité est l'endroit le plus en aval du Saguenay, où nous avons observé un grand nombre de cailloux de dolomie sur une batture argileuse bordée d'une falaise en matériel meuble (Dionne 2001).

Un inventaire des cailloux de dolomie sur le rivage compris entre Les Escoumins et Baie-des-Bacons a été réalisé au cours des dernières années afin de mieux connaître la répartition géographique de ce type de lithologie et d'obtenir des renseignements utiles pour reconstituer certains grands écoulements glaciaires au Wisconsinien (Dionne et Bernatchez 2000).

\section{Caractéristiques du Milieu}

Le secteur côtier, entre Les Escoumins et Baie-desBacons (Fig. 1), est caractérisé par de grandes terrasses constituées de dépôts meubles quaternaires. Ces terrasses, qui s'élèvent jusqu'à 145-150 m d'altitude, correspondent à la zone submergée par la Mer de Goldthwait (Dionne 1977) et forment une plaine côtière de quelques dizaines de kilomètres de largeur.

Le littoral actuel comprend plusieurs secteurs composés de roches précambriennes, principalement des gneiss variés, de la pegmatite, des amphibolites et leptinites, ainsi que des quartzites (Rondot 1986). Le modelé glaciaire est évident partout; le dernier écoulement est à $60^{\circ} \mathrm{NE}$. Cependant, $80 \%$ du trait de côte est composé de dépôts meubles fini-glaciaires et holocènes entaillés en une falaise de 20 à $30 \mathrm{~m}$ de hauteur (Fig. 2a), au pied de laquelle s'étend une vaste batture argileuse, de 1 à $2 \mathrm{~km}$ de largeur. Au pied de la falaise, on trouve une étroite bande ( $10 \mathrm{a} 25 \mathrm{~m}$ de largeur) de sable et gravier correspondant à un haut de plage, qui est caractérisé par de très nombreux blocs et de gros galets (Fig. 2b). En pente faible et quasi-horizontale, la batture adjacente est, elle aussi, parsemée de méga-blocs épars et de dallages de petits blocs (Fig. 2c-d). Il s'agit d'une plate-forme d'érosion taillée dans les dépôts de la terrasse de $20-30 \mathrm{~m}$, au cours de l'Holocène.

En général, la terrasse de $20-30 \mathrm{~m}$ comprend quatre unités lithostratigraphiques (Dionne et Occhietti 1996, Fig. 5, p. 10). À la base de la falaise et sur la batture, on observe une argile marine brunâtre non datée, contenant entre 15 et $20 \%$ de carbonates, qui est propre au secteur de la rive nord, à l'est de Tadoussac. L'analyse de la micro-faune d'un échantillon provenant du secteur 4 des Escoumins a révélé la présence d'une quinzaine d'espèces de foraminiferes et quelques ostracodes (Guilbault, 2000). La faune est toutefois dominée par quatre espèces de foraminifères: Cassidulina reniforme (61\%), Elphidium excavatum forma clavata (17\%), Islandiella helenae (13\%) et Stainfortia feylingi (7\%). Selon Guilbault, cette faune serait caractéristique de la phase 1 de la Mer de Goldthwait (Dionne 1977).

Au-dessus de ce dépôt, argileux, il y a un diamicton gris sableux et argileux, d'épaisseur variable mais en général de 3 à $4 \mathrm{~m}$. Cette unité est surmontée par une séquence stratifiée de limon/argile gris, calcaire et fossilifêre, de 6-7 m d'épaisseur, daté à $10,7 \mathrm{ka}$, à Grandes-Bergeronnes. Au sommet, on observe une couche d'environ deux mètres de sable et petit gravier, jaunâtre à brunâtre selon le degré d'oxydation, qui correspond à des plages.

À la sortie est du village des Escoumins, une coupe plus complexe montre trois unités argileuses, deux unités de diamicton et deux unités sableuses (Fig. 3). Par endroits, les rythmites limono-argileuses dans la partie supérieure de la coupe contiennent de rares valves de Portlandia arctica et Macoma balthica. L'unité argileuse en dessus du premier diamicton a donné un âge radiocarbone de 10,250 BP (UL1921) sur Macoma calcarea. Cette coupe complexe n'est toutefois pas représentatiave de l'ensemble du secteur étudié; elle indique plutôt l'existence de variations latérales des unités lithostratigraphiques de la terrasse de 20-30 m.

\section{Observations}

Un inventaire des cailloux de dolomie, principalement des blocs, mais aussi des gros galets, a été fait principalement sur 

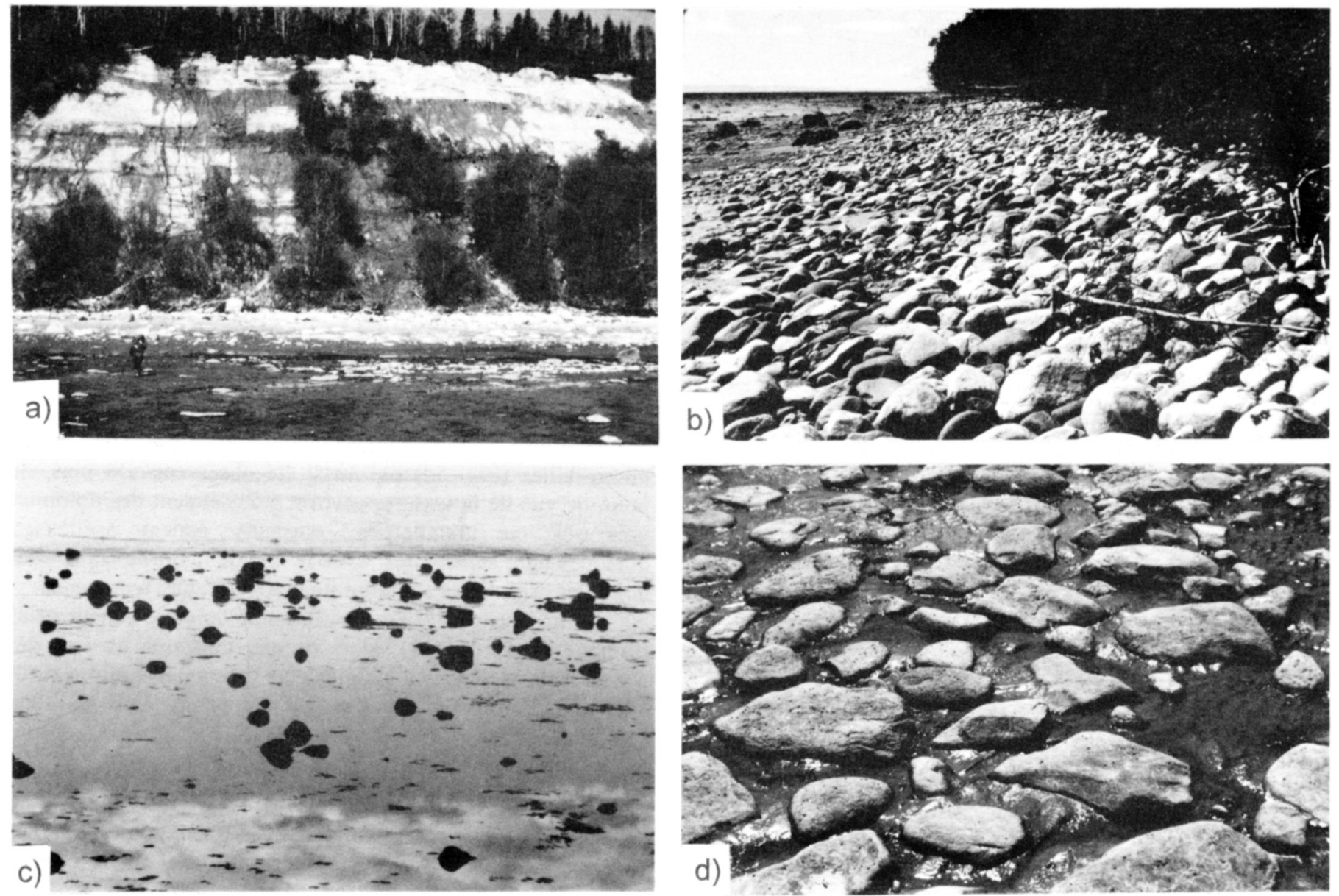

Fig. 2. a) Falaise vive dans la terrasse de $30 \mathrm{~m}$ dans le secteur 3 , à l'ouest de la rivière Petits-Escoumins; à remarquer les blocs résiduels au pied de la falaise et un petit dallage de cailloux à droite du personnage dans la partie supérieure de la batture argileuse (4-6-97). b) Vue typique du haut du rivage au pied de la falaise dans le secteur 6; montrant des blocs de petite à moyenne taille bien lessivés formant un cordon de 15 à $20 \mathrm{~m}$ de largeur (24-6-98). c) Vue générale de la batture argileuse à méga-blocs épars juste à l'est du village des Escoumins (24-6-98). d) Un dallage de cailloux typique de la batture argileuse dans la région des Escoumins (20-05-00).

le haut du rivage, soit une zone de 15 à $20 \mathrm{~m}$ de largeur au pied de la falaise. Tous les cailloux de dolomie dans le secteur étudié n'ont pas forcément été observés.

Le littoral inventorié s'étend sur $18 \mathrm{~km}$ de longueur. Pour des raisons pratiques, nous l'avons subdivisé en six soussecteurs d'inégale longueur. Le secteur 1 , juste à l'est du village des Escoumins, mesure environ $1,8 \mathrm{~km}$ de longueur; ceux des anses à Thibault et à Pelletier (secteurs 2 et 3), ainsi que celui à l'ouest de l'embouchure de la rivière des PetitsEscoumins (secteur 4) mesurent environ $2 \mathrm{~km}$ chacun; le cinquième secteur, d'environ $6,2 \mathrm{~km}$, est compris entre la rivière des Petits-Escoumins et la rivière Petite-Romaine, alors que le sixième, de $4 \mathrm{~km}$, s'étend entre la rivière PetiteRomaine et Baie-des-Bacons.

Les diverses caractéristiques des cailloux ont été notées, notamment la couleur, la forme, le façonnement; on a aussi mesuré les trois axes de manière à pouvoir estimer le poids. Dans le calcul du poids, on a tenu compte de la forme des cailloux et, selon le cas, réduit la valeur obtenue de 10,25 ou $40 \%$. Il s'agit bien entendu d'une estimation. En général, le poids obtenu est inférieur au poids réel. Cette information nous est apparue utile pour évaluer le transport de matériel grossier sur de longues distances (Prest and Nielsen 1987).

\section{Caractéristiques des Cailloux}

À l'instar du secteur à l'embouchure du Saguenay (Dionne 1994), plusieurs variétés de dolomie ont été observées dans la région des Escoumins. L'inventaire réalisé porte sur le nombre de cailloux par secteur, la couleur, le faciès, la présence de stries et autres particularités, la taille, le degré de façonnement, ainsi que sur la présence de d'autres lithologies particulières pouvant servir $d^{\prime}$ 'indicateurs.

\section{Nombre de cailloux}

Le Tableau 1 indique le nombre de cailloux de dolomie par secteur et pour l'ensemble de la zone étudiée. Au total, 3661 cailloux de dolomie ont été observés, décrits et mesurés. Compte tenu de leur longueur ( $2 \mathrm{~km}$ environ), les secteurs 2 et 3 contiennent davantage (54\%) d'erratiques de dolomie que les quatre autres. La répartition des cailloux de dolomie est donc inégale sur l'ensemble du secteur étudié, ce qui reflète sans doute des points de meilleure alimentation.

\section{Couleur des cailloux}

Une des caractéristiques principales est la couleur de la roche. Le Tableau 2 indique les pourcentages par secteurs et 


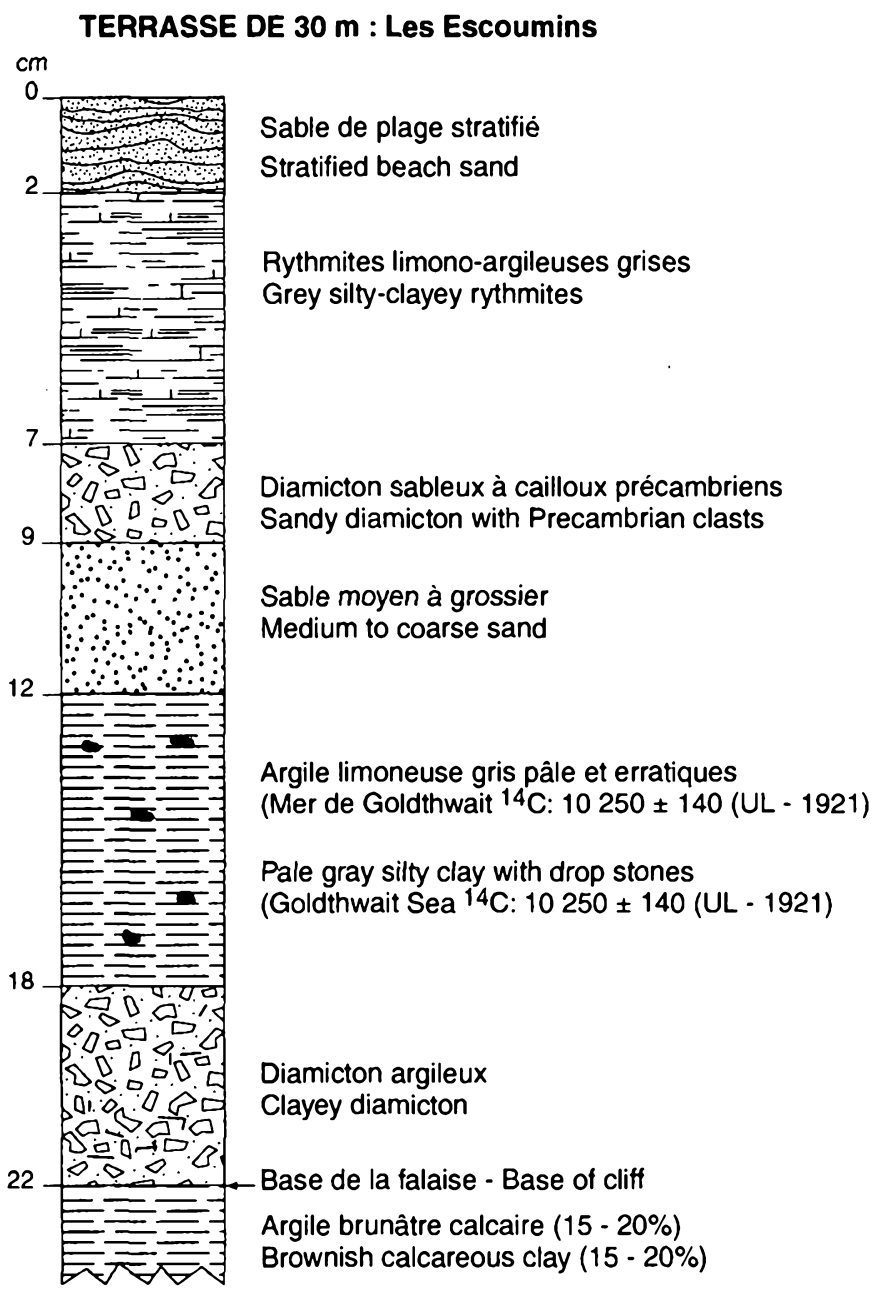

Fig. 3. Coupe lithostratigraphique de la terrasse de $30 \mathrm{~m}$, à l'est du village des Escoumins.

pour l'ensemble des différentes variétés. On constate facilement que les cailloux de dolomie grise (Fig. 4a) dominent largement avec près de $74 \%$. L'autre catégorie la plus fréquente ( $24 \%$ environ), comprend des dolomies rose pâle à rose foncé (Fig. 4b), roses tachées de gris (Fig. 4c), gris rosâtre ou rougeâtre, roses et rouges ainsi que des dolos rouges. Le reste $(2 \%)$ est composé de dolomies blanches, brunes ou noires. Il s'agit, bien entendu, de la couleur en coupe fraîche et non de la couleur de la surface des cailloux qui, dans 80 à $85 \%$ des cas, était plus pâle ou légèrement différente en raison de l'altération superficielle.

\section{Autres caractéristiques}

D'autres caractéristiques (Tableau 3) ont permis de distinguer plusieurs variétés de dolomie. Ainsi, certains cailloux de dolomie grise et une rose contenaient des stromatolites (Fig. 4d). Au total, 30 cailloux de cette catégorie ont été observés. D'autres dolos, généralement de couleur grise étaient soit marbrées, soit rubanées ou laminées (Fig. 4e) avec parfois des micro-plis ou des déformations (Fig. 4f). Environ $8 \%$ contenaient des inclusions de quartz ou de chert noir (Fig. 5a). Plus de 3\% étaient bréchiques ou conglomératiques (Fig. 5b). Plus de 10\% des cailloux de dolomie observés provenaient de formations stratifiées (Fig.
Tableau 1. Nombre de cailloux de dolomie observés dans le secteur entre les Escoumins et Baie-des-Bacons

\begin{tabular}{|c|c|c|c|}
\hline Secteur & Total & Blocs & Galets \\
\hline 1. Escoumins Village & 151 & 120 & 31 \\
\hline 2. Anse à Thibault & 1166 & 909 & 257 \\
\hline 3. Anse à Pelletier & 822 & 693 & 129 \\
\hline 4. Petits-Escoumins-Ouest & 203 & 175 & 28 \\
\hline 5. Petits-Escoumins-Est & 662 & 662 & - \\
\hline 6. Petite-Romaine-Est & 657 & 632 & 25 \\
\hline Total & 3661 & 3191 & 470 \\
\hline
\end{tabular}

5c). D'autres caractéristiques comme des stylolites et des micro-failles (Fig. 5d) ont aussi été observées. De plus, du point de vue de la texture, environ 5,5\% étaient des dolomies gréseuses ou granuleuses; plusieurs étaient argileuses, quelques-unes schisteuses, mais la grande majorité étaient des dolomies cristallines à texture fine (aphanitique) à moyenne.

Enfin, plus de $28 \%$ des cailloux de dolomie étaient striés (Fig. 5e et Tableau 4), alors qu'une douzaine avaient des fractures de broutage et que plusieurs gros galets polis et striés avaient la forme typique " en fer à repasser » des cailloux glaciaires.

\section{Façonnement et taille des cailloux}

Concernant le façonnement et l'usure, plus de $72 \%$ des cailloux de dolomie mesurés tombent dans les catégories subanguleux à subarrondis et subarrondis (Tableau 5). II y a très peu (3\%) de cailloux anguleux et arrondis.

La taille des cailloux est une caractéristique importante à connaître dans le cas d'un transport glaciaire sur de longues distances et renseigne utilement sur l'étendue des affleurements. La plupart des auteurs reconnaissent que la quantité ou le volume des éléments grossiers (blocs) chute rapidement au-delà de $30-35 \mathrm{~km}$ de la source. C'est pourquoi nous avons mesuré les cailloux de dolomie du rivage des Escoumins.

Ont été considérés ici uniquement les cailloux ayant un poids supérieur à un kilo, soit un total de 3191 cailloux. Dans ce groupe, on constate une nette prédominance des petits cailloux, ceux de 1 à $5 \mathrm{~kg}$ comptant pour plus de $40 \%$. Les gros blocs ne représentent que $11 \%$, dont $4 \%$ de cailloux pesant plus de $100 \mathrm{~kg}$ (Tableau 6). Le Tableau 7 indique la taille et le poids des plus gros cailloux de dolomie observés. Il y en a deux d'environ une tonne et un de deux tonnes. Le Tableau 8 indique les dimensions des cailloux de dolomie à stromatolites, car il s'agit du meilleur indicateur observé sur le rivage des Escoumins. Les deux plus gros blocs pesaient respectivement 280 et 175 kilos.

\section{Autres lithologies}

Parmi les autres lithologies exotiques, d'origine lointaine, on a trouvé plusieurs blocs d'anorthosite dont certains de taille métrique; quelques dizaines de cailloux de calcaire fossilifêre de Trenton (Ordovicien); beaucoup de quartzites blancs ou roses, et quelques roches ferrifères; deux blocs de conglomérat à clastes calcaires apparemment appalachiens et quelques 
Tableau 2. Couleur des cailloux de dolomie observés dans le secteur entre Les Escoumins et Baie-des-Bacons

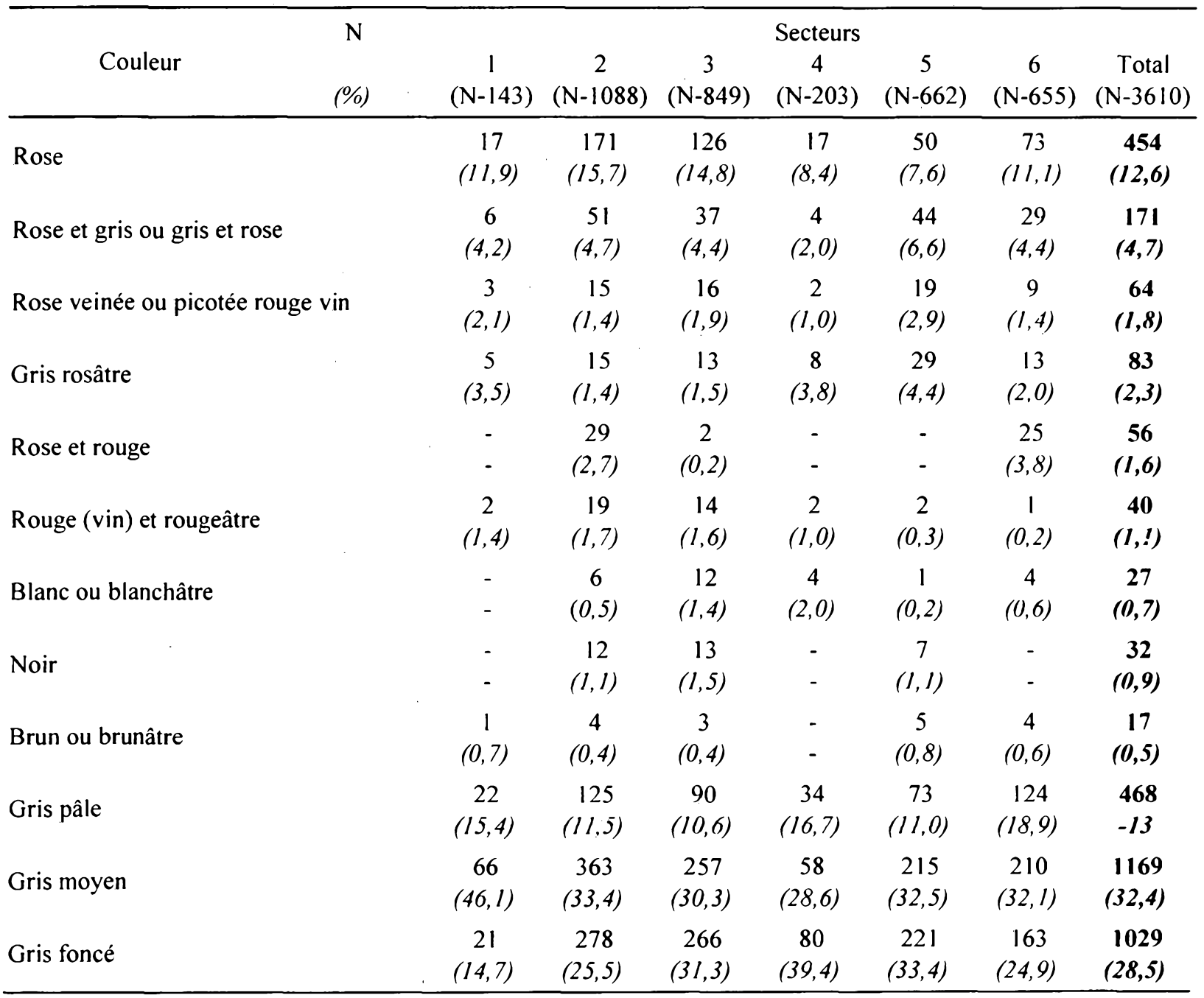

autres à galets de quartz ainsi que deux blocs de schistes appalachiens, l'un gris, l'autre rouge. Un seul bloc de tillite (Fig. 5f) a été observé dans le secteur des Escoumins, alors que nous en avions trouvé une dizaine à l'embouchure du Saguenay (Dionne 1994).

\section{Origine des Cailloux de Dolomie}

L'absence de formations de dolomie dans la région des Escoumins (Rondot 1986) confêre aux erratiques de cette lithologie une valeur comme indicateur à la fois d'un transport par les glaciers, les icebergs et les glaces flottantes.

\section{Les formations de roches dolomitiques au Québec}

A-Les dolomies à stromatolites

Selon Hans J. Hoffmann (communication personnelle), spécialiste des dolomies à stromatolites, on connaît seulement cinq sites de formations dolomitiques à stromatolites au Québec: trois d'âge Protérozoïque et deux d'âge Ordovicien inférieur. Des deux derniers, l'un se trouve dans la formation de Beekmantown, dans la région de Montréal (Clark 1972), et l'autre, dans la formation de Romaine, dans la région de Mingan; à cet endroit, cependant, il s'agit surtout de calcaires dolomitiques et, d'après Twenhofel (1938) et Waddington (1950), les structures stromatolitiques sont peu ou mal développées.

Quant aux dolomies à stromatolites du Protérozoïque, elles affleurent sur de grandes étendues sur la côte québécoise de la mer d'Hudson, entre la Grande île et Inukjuak. Il en existe aussi dans le bassin sédimentaire de Mistassini (Neilson 1953; Wahl 1953; Caty 1976) ainsi que dans le fossé du Labrador (Donaldson 1963, 1966; Baragar 1967; Dimroth 1978; Dresser 1979). Dans ces deux derniers endroits, on trouve aussi plusieurs variétés de dolomie, dont certaines sont roses, d'autres rouges, blanches, noires et verdâtres.

Malheureusement, il existe peu d'études détaillées sur ces divers types de dolomie $\mathrm{y}$ compris les dolomies à stromatolites. Selon Hans J. Hoffmann (1969, 1973), les espèces de cryptozoaires (blue-green algae) à l'origine des structures stromatolitiques ne sont pas connues, seules la forme des édifices a été étudiée. Quoi qu'il en soit, l'aspect des dolomies à stromatolites du Protérozoïque differe suffisamment de celle de l'Ordovicien pour permettre de les distinguer assez facilement, lorsqu'on les connaît.

Par rapport aux Escoumins, les trois aires de dolomies du Protérozoïque sont situées respectivement entre 400 et $500 \mathrm{~km}$ 

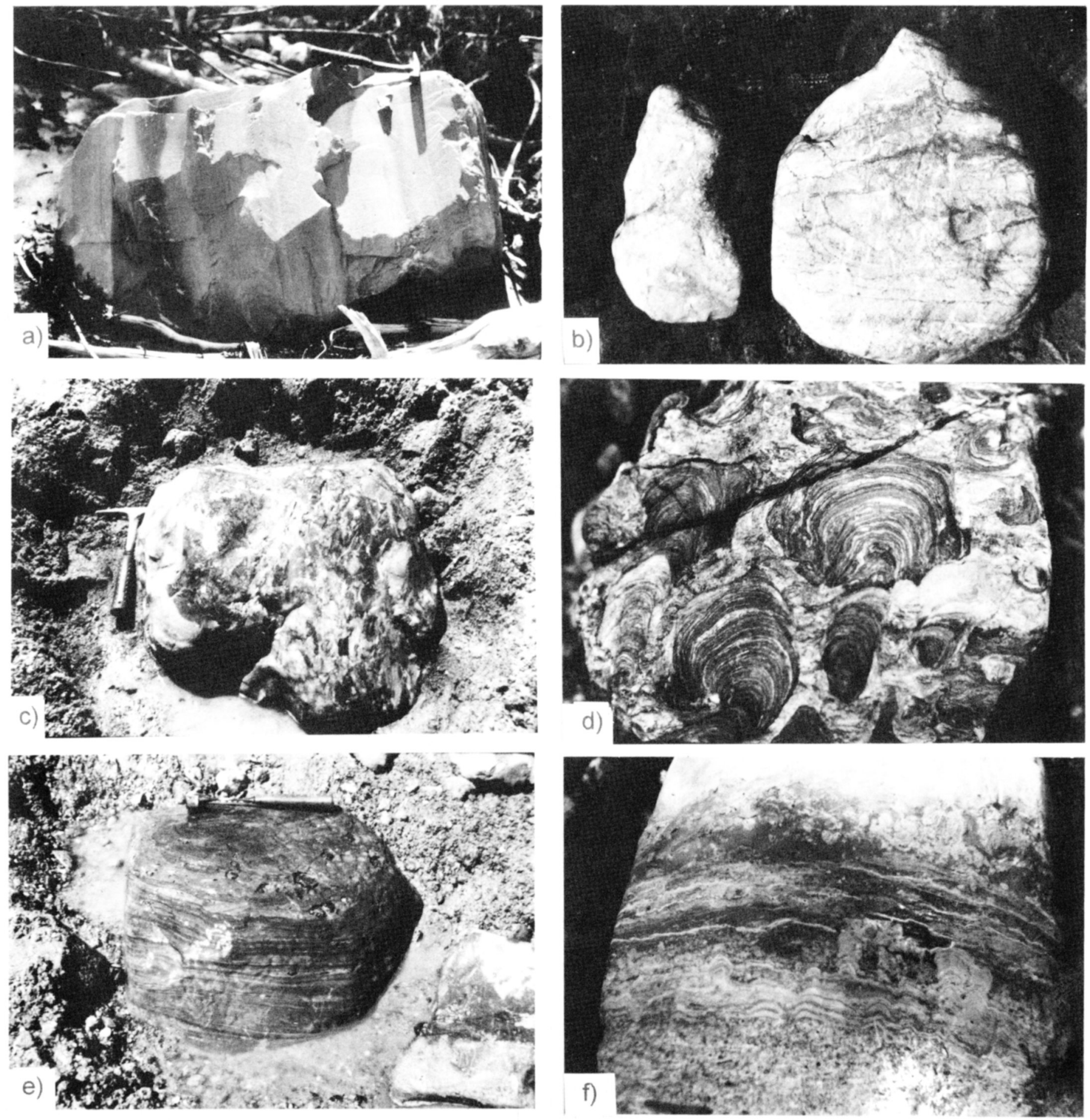

Fig. 4. a) Bloc erratique de dolomie gris moyen à foncé altéré chamois sur le haut du rivage au pied de la falaise dans le secteur 3; le bloc mesure $105 \times 65 \times 65 \mathrm{~cm}$ et pèse environ $832 \mathrm{~kg}(20-05-00)$. b) Blocs de dolomie de couleur rose; le plus gros avec des lamines alternées rose pâle, rose foncé et rouge vin mesure $36 \times 30 \times 12 \mathrm{~cm}$ et pèse environ $24 \mathrm{~kg}$; le petit bloc contient des inclusions de quartz (20-5-00). c) Bloc de dolomie rose tachetée de gris, un type relativement fréquent, observé sur la plage dans le secteur 2 ; le bloc mesure $60 \times 52 \times 33 \mathrm{~cm}$ et pèse environ $193 \mathrm{~kg}$ (20-5-00). d) Un petit bloc de dolomie à stromatolites, d'environ $12 \mathrm{~kg}$, provenant des formations d'âge protérozoïque du bassin sédimentaire de Mistassini, observé dans le secteur 2 (26-6-99). e) Bloc erratique de dolomie laminé gris foncé, un type fréquent, partiellement enfoui dans le sable de la plage du secteur 2; le bloc mesure $53 \times 45 \times 31 \mathrm{~cm}$ et pèse environ $38 \mathrm{~kg}(20-5-00)$. f) Bloc de dolomie laminé gris moyen avec lamines ondulées (stromatolites) et lits bréchiques observé dans le secteur 3 ; le caillou mesure $36 \times 36 \times 214 \mathrm{~cm}$ et pèse environ 70 $\mathrm{kg}(4-6-97)$. 
Tableau 3. Caractéristiques des cailloux de dolomie observés dans le secteur entre Les Escoumins et Baie-des-Bacons

\begin{tabular}{lccccccc}
\hline \multicolumn{1}{c}{ Caractéristiques } & \multicolumn{9}{c}{ Nombre de dolo par secteurs } & \multicolumn{2}{c}{ Total } \\
& 1 & 2 & 3 & 4 & 5 & 6 & (3) \\
& $(151)$ & $(1166)$ & $(882)$ & $(203)$ & $(662)$ & $(657)$ & $(3661)$ \\
\hline À stromatolites & - & 8 & 13 & - & 2 & 7 & $\mathbf{3 0}$ \\
Type rubané-laminé (généralement gris) & $\mathbf{8}$ & 21 & 21 & 6 & 13 & 5 & $\mathbf{7 4}$ \\
Type marbré & 6 & 32 & 25 & 17 & 53 & 13 & $\mathbf{1 4 6}$ \\
Type protérozoïque gris foncé avec quartz & 1 & 4 & 9 & 2 & 11 & 9 & $\mathbf{3 6}$ \\
Avec quartz & 11 & 53 & 61 & 35 & 78 & 65 & $\mathbf{3 0 3}$ \\
Avec chert noir & - & 1 & 3 & - & 4 & 5 & $\mathbf{1 3}$ \\
Type bréchique ou conglomératique & 4 & 29 & 24 & 13 & 32 & 21 & $\mathbf{1 2 3}$ \\
Type stratifié & 18 & 86 & 98 & 24 & 88 & 82 & $\mathbf{3 9 6}$ \\
Gréseux/granuleux & 12 & 49 & 47 & 4 & 46 & 45 & $\mathbf{2 0 3}$ \\
Schisteuse & 6 & 2 & 3 & - & 1 & - & $\mathbf{1 2}$ \\
Formes de corrosion et/ou dissolution & - & - & 10 & - & - & - & $\mathbf{1 0}$ \\
Fractures de broutage & - & 1 & 5 & 1 & 1 & 4 & $\mathbf{1 2}$ \\
Turbations ou lits ondulés & - & - & 1 & - & 6 & 1 & $\mathbf{8}$ \\
Micro-failles & - & - & 2 & 1 & 2 & - & $\mathbf{5}$ \\
\hline
\end{tabular}

Tableau 4. Nombre de cailloux de dolomie striés observés dans le secteur entre Les Escoumins et Baie-des-Bacons

\begin{tabular}{|c|c|c|c|}
\hline Secteur & $\begin{array}{l}\text { Nombre total de } \\
\text { cailloux }\end{array}$ & N-striés & $\%$ striés \\
\hline 1 & 151 & 48 & 31,8 \\
\hline 2 & 1166 & 444 & 38,0 \\
\hline 3 & 822 & 227 & 27,6 \\
\hline 4 & 203 & 31 & 15,2 \\
\hline 5 & 662 & 211 & 31,9 \\
\hline \multirow[t]{2}{*}{6} & 657 & 86 & 13,1 \\
\hline & Total & 1031 & $\overline{28,2}$ \\
\hline
\end{tabular}

Tableau 6. Poids des cailloux' de dolomie par catégories (en pourcentage)

\begin{tabular}{cccccccc}
\hline & \multicolumn{7}{c}{ Secteur } \\
Catégories & 1 & 2 & 3 & 4 & 5 & 6 & Moyenne \\
\hline 1 à $5 \mathrm{~kg}$ & 47,4 & 46,2 & 48,7 & 37,4 & 30,4 & 33,5 & $\mathbf{4 0 , 6}$ \\
5 à $10 \mathrm{~kg}$ & 15,2 & 20,7 & 17,0 & 17,8 & 16,8 & 23,9 & $\mathbf{1 8 , 6}$ \\
10 à $20 \mathrm{~kg}$ & 13,6 & 17,0 & 13,0 & 12,6 & 20,2 & 20,3 & $\mathbf{1 6 , 1}$ \\
20 à $50 \mathrm{~kg}$ & 10,2 & 9,8 & 13,4 & 13,2 & 20,7 & 14,2 & $\mathbf{1 3 , 6}$ \\
50 à $100 \mathrm{~kg}$ & 10,2 & 4,3 & 4,5 & 9,8 & 7,2 & 5,3 & $\mathbf{6 , 9}$ \\
$>100 \mathrm{~kg}$ & 3,4 & 2,0 & 3,3 & 9,2 & 4,6 & 2,7 & $\mathbf{4 , 2}$ \\
\hline
\end{tabular}

'Cailloux de $1 \mathrm{~kg}$ et plus seulement; galets de $<1 \mathrm{~kg}$ non compris

pour le bassin sédimentaire de Mistassini, 750 à $900 \mathrm{~km}$ pour le fossé du Labrador, alors que la côte est de la mer d'Hudson est à plus de $1000 \mathrm{~km}$.

\section{B - Formations de dolomie sans stromatolites}

Les cartes géologiques disponibles du Bouclier Laurentidien, au nord des Escoumins (Rondot 1986) renseignent peu sur l'existence de formations dolomitiques dans le Grenville. Seul Avramtchev (1985) signale l'existence de deux petits affleurements de dolomie au NO des
Tableau 5. Émoussé visuel des cailloux de dolomie observés dans le secteur entre Les Escoumins et Baie-des-Bacons

\begin{tabular}{cccc}
\hline Façonnement (émoussé) & $\begin{array}{c}\mathrm{N}-3191 \\
\text { Blocs \% }\end{array}$ & $\begin{array}{c}\mathrm{N}-470 \\
\text { Galets \% }\end{array}$ & $\begin{array}{c}\mathrm{N}-3661 \\
\text { Cailloux \% } \\
\text { Total }\end{array}$ \\
\hline Anguleux (AN) & 1,4 & 0,5 & $\mathbf{1 , 1}$ \\
Anguleux-subanguleux (AN-SAN) & 1,85 & 0,1 & $\mathbf{1 , 1 5}$ \\
Subanguleux (SAN) & 15,2 & 12,2 & $\mathbf{1 4}$ \\
Subanguleux-subarrondi (SAN-SAR) & 23,7 & 20 & $\mathbf{2 2 , 5}$ \\
Subarrondi (SAR) & $\mathbf{4 6 , 0}$ & 55,6 & $\mathbf{4 9 , 8}$ \\
Subarrondi-arrondi (SAR-AR) & 10,3 & 8,5 & $\mathbf{9 , 5}$ \\
Arrondi (AR) & 1,5 & 3,2 & $\mathbf{1 , 8}$ \\
AN/AN-SAN/SAN & 18,45 & 12,8 & 15,6 \\
SAN-SAR/SAR & 69,7 & 75,6 & $\mathbf{7 2 , 6 5}$ \\
SAR-AR/AR & 11,8 & 11,7 & $\mathbf{1 1 , 7 5}$ \\
\hline
\end{tabular}

Escoumins. Mais les cartes géologiques du Grenville (Franconi et al. 1975; Laurin et Sharma 1975) et la carte détaillée de Greig (1952) ne mentionnent pas ce site. Par ailleurs, la carte d'Avramtchev (1985) indique une aire relativement grande de marbre et de dolomie dans le Grenville entre Hull et le lac Bastong, dans le SO du Québec, ainsi que trois petits affleurements au SO de la cuvette du Lac SaintJean $\left(48^{\circ} 10^{\prime} \mathrm{N}, 73^{\circ} \mathrm{O}\right)$.

Les formations appalachiennes au sud du Saint-Laurent estuarien contiennent parfois des lits de dolomie. Toutefois, les mentions et les descriptions sont plutôt laconiques; on connaît donc mal l'importance de cette lithologie. La dolomie se rencontre principalement dans le Silurien et le Dévonien et rarement dans les formations de la bande côtière d'une vingtaine de kilomètres de largeur, d'âge Cambro-Ordovicien (Slivitsky et al. 1991). La carte géo-touristique du Bas-SaintLaurent et de la Gaspésie (Tremblay et Bourque 1991) par exemple, ne mentionne aucun site de dolomie. Bref, les connaissances sur les formations de dolomie au Québec sont 

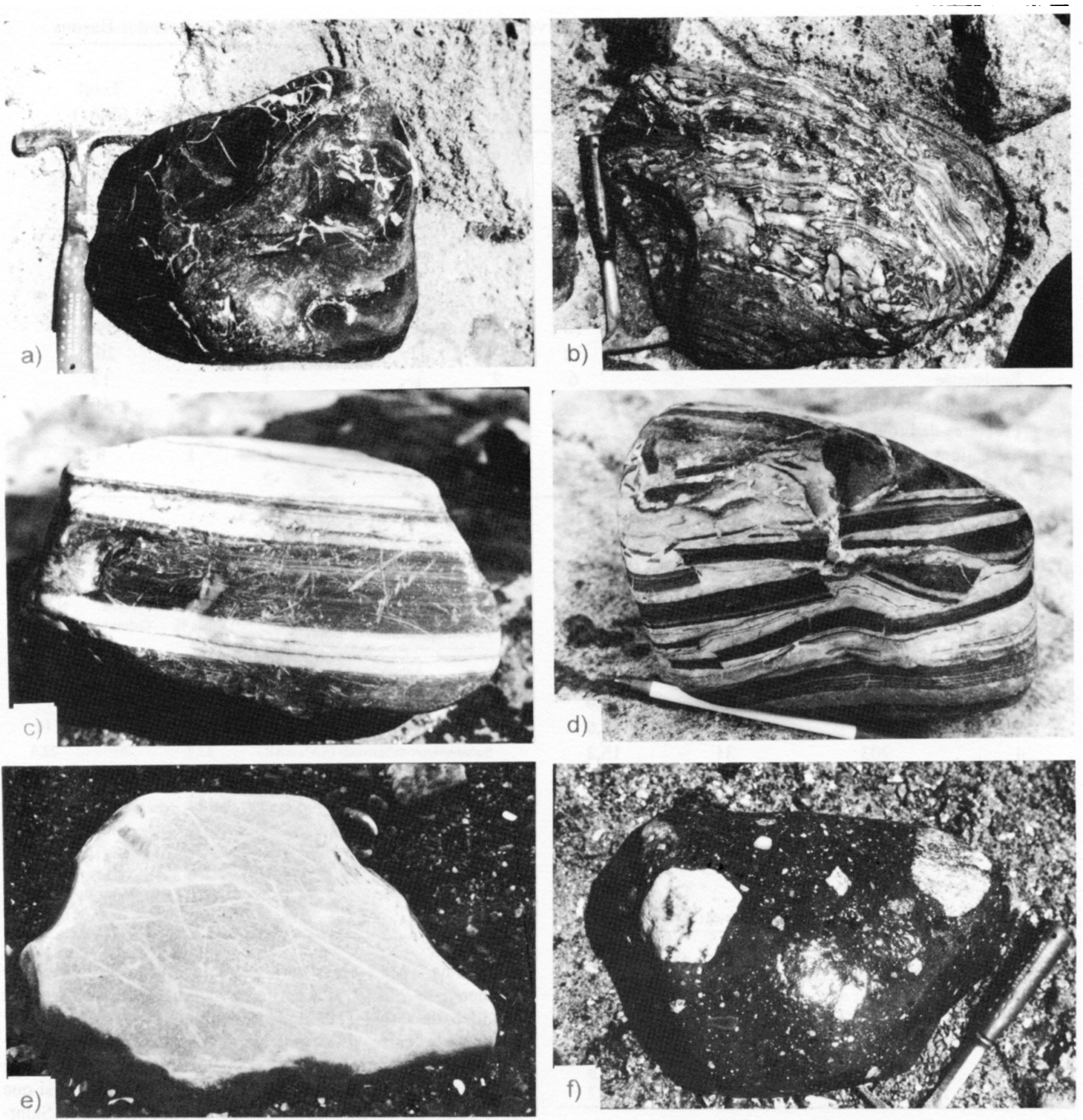

Fig. 5. a) Bloc de dolomie gris foncé avec inclusions de quartz, un faciès typique de certaines couches des formations protérozoïques du bassin sédimentaire de Mistassini; le bloc mesure $30 \times 28 \times 10 \mathrm{~cm}$ et pèse environ $19 \mathrm{~kg}$ (23-6-98). b) Bloc de dolomie bréchique gris moyen à foncé, un type relativement fréquent dans la région des Escoumins; le bloc mesure $50 \times 34 \times 23 \mathrm{~cm}$ et pèse environ $73 \mathrm{~kg}(27.6 .99)$. c) Bloc de dolomie de type stratifié montrant une alternance de couches gris foncé et blanches; le bloc est strié et mesure $26 \times 20 \times 12 \mathrm{~cm}$ et pèse environ $12 \mathrm{~kg}$ (266-98). d) Bloc de dolomie stratifié avec couches faillées alternées gris pâle et gris foncé; le bloc mesure $20 \times 15 \times 10 \mathrm{~cm}$ et pèse environ $5,6 \mathrm{~kg}$ (24-6-98). e) Bloc de dolomie strié gris moyen altéré plus pâle; le caillou mesure $38 \times 28 \times 12 \mathrm{~cm}$ et pèse environ $24 \mathrm{~kg}(27-6-99)$. f) Bloc de tillite avec clastes ignées et métamorphiques provenant probablement de la formation du lac Waconichi, au NE de Chibougamau; le bloc mesure $36 \times 24 \times 20 \mathrm{~cm}$ et pèse environ $32 \mathrm{~kg}(27-6-99)$. 
Tableau 7. Les dix plus gros blocs de dolomie observés dans le secteur entre Les Escoumins et Baie-des-Bacons

\begin{tabular}{|c|c|c|c|}
\hline Dimensions $(\mathrm{cm})$ & Poids (kg) & Secteur & Nature \\
\hline $125 \times 115 \times 75$ & 2000 & 2 & Dolo cristalline gris divers - rubanée \\
\hline 2. $120 \times 90 \times 50$ & 1000 & 3 & Dolo rouge vin altérée beige - stratifiée \\
\hline 3. $125 \times 90 \times>40$ & 1000 & 3 & Dolo cristalline gris moyen - corrodée \\
\hline 4. $100 \times 95 \times 52$ & 926 & 5 & Dolo gris moyen altérée beige - stratifiée et striée \\
\hline 5. $105 \times 65 \times 65$ & 832 & 4 & Dolo gris foncé altérée beige - striée \\
\hline 6. $115 \times 70 \times 40$ & 604 & 4 & Dolo cristalline rose altérée crème \\
\hline 7. $92 \times 72 \times 48$ & 596 & 6 & Dolo gris foncé avec quartz - type protérozoïque \\
\hline 8. $125 \times 85 \times 28$ & 558 & 3 & Dolo gris divers - bréchique \\
\hline 9. $80 \times 75 \times 40$ & 540 & 3 & Dolo gris divers rubanée - corrodée et striée \\
\hline 10. $98 \times 70 \times 30$ & 463 & 2 & Dolo gris divers conglomératique \\
\hline
\end{tabular}

Tableau 8. Cailloux de dolomie à stromatolites observés dans le secteur entre Les Escoumins et Baie-des-Bacons

\begin{tabular}{lcccc}
\hline $\begin{array}{c}\text { Dimensions } \\
(\mathrm{cm})\end{array}$ & $\begin{array}{c}\text { Poids } \\
(\mathrm{kg})\end{array}$ & Émoussé & Type & Secteur \\
\hline $30 \times 25 \times 17$ & 24,0 & SAR & A & 2 \\
$21 \times 20 \times 20$ & 19,0 & SAN & A & 2 \\
$21 \times 19 \times 15$ & 11,5 & SAN & A & 2 \\
$20 \times 12 \times 10$ & 4,5 & SAN & A & 2 \\
$17 \times 14 \times 9$ & 4,0 & SAR & A & 2 \\
$17 \times 13 \times 8$ & 3,5 & SAN-SAR & A & 2 \\
$16 \times 13 \times 9$ & 3,5 & SAN-SAR & A & 2 \\
1 galet $-6 \mathrm{~cm}$ & - & SAN-SAR & A & 2 \\
$70 \times 40 \times 25$ & 175,0 & AN & A & 3 \\
$42 \times 29 \times 28$ & 64,0 & AN & A & 3 \\
$35 \times 32 \times 25$ & 58,0 & SAR & A & 3 \\
$35 \times 28 \times 27$ & 50,0 & SAN & A & 3 \\
$27 \times 27 \times 22$ & 30,0 & SAN & B & 3 \\
$36 \times 24 \times 18$ & 29,2 & SAR & B & 3 \\
$24 \times 23 \times 16$ & 19,9 & SAN & B & 3 \\
$20 \times 15 \times 13$ & 7,3 & SAR & A & 3 \\
$17 \times 15 \times 12$ & 6,9 & SAN & A & 3 \\
$16 \times 15 \times 10$ & 5,4 & AN & A & 3 \\
$11 \times 9 \times 6$ & 1,1 & SAR & B & 3 \\
1 galet -9 cm & - & SAN & A & 3 \\
1 galet $-8 \mathrm{~cm}$ & - & SAN & A & 3 \\
$30 \times 30 \times 22$ & 37,0 & SAR-AR & B & 5 \\
$15 \times 10 \times 8$ & 2,2 & AR & A & 5 \\
$60 \times 52 \times 40$ & 280,0 & SAN-SAR & A & 6 \\
$45 \times 35 \times 32$ & 113,0 & SAR & A & 6 \\
$35 \times 30 \times 25$ & 49,5 & SAR & A & 6 \\
$35 \times 32 \times 16$ & 34,0 & SAR & A & 6 \\
$32 \times 26 \times 12$ & 26,0 & SAR & A & 6 \\
$22 \times 18 \times 14$ & 10,4 & SAR & A & 6 \\
$26 \times 20 \times 10$ & 10,0 & SAR & C & 6 \\
1 galet $-8 \mathrm{~cm}$ & - & SAR & A & 6 \\
\hline
\end{tabular}

Type A : Dolo gris foncé avec quartz - type protérozoïque

Type $\mathrm{B}$ : Dolo gris pale à moyen - rubanée/laminée

Type C : Dolo rose loin d'être satisfaisantes, d'où la difficulté de retracer la source des erratiques des Escoumins et des autres régions.

\section{La source des cailloux de dolomie des Escoumins}

D'où proviennent les divers cailloux de dolomie du rivage entre Les Escoumins et Baie-des-Bacons? Il est difficile de répondre à cette question d'une manière pleinement satisfaisante en raison d'une méconnaissance des formations dolomitiques dans le Bouclier Laurentidien. Toutefois, certains faciès, notamment celui à stromatolites, portent à penser que les erratiques de dolomie du rivage des Escoumins ont une source commune, qui, dans l'état actuel des connaissances, ne peut être que le bassin sédimentaire d'âge Protétozoïque de Mistassini.

Pourquoi? Premièrement parce qu'on y a signalé des faciès dolomitiques semblables à ceux observés aux Escoumins (Neilson 1953; Wahl 1953); deuxièmement, parce que l'existence d'un écoulement glaciaire du NO vers le SE à partir d'un dôme glaciaire situé au NO du lac Mistassini a été établie au cours des deux dernières décennies (Boulton et al. 1985; Fisher et al. 1985; Veillette 1986; Dyke et Prest 1987a, 1987b; Parent et al. 1995, Veillette et al. 1999); troisièmement, parce que les trois autres aires où il existe des formations de dolomie (côte est de la mer d'Hudson, fossé du Labrador et région au nord de Hull) sont situées trop loin et en dehors des axes d'écoulement glaciaire, au Wisconsinien, qui ont atteint l'estuaire du Saint-Laurent (Veillette et al. 1999 Occhietti et al., 2001).

Par ailleurs, nous éliminons d'emblée la rive sud de l'estuaire (région des Appalaches) comme source possible des erratiques de dolomie du rivage des Escoumins sachant 1) que les déplacements de cailloux par les glaces flottantes de la rive sud à la rive nord sont exceptionnels en raison principalement des courants (Dionne 1972, 1994); 2) que la glace appalachienne, qui a atteint la rive sud lors de la déglaciation, n'a pas débordé très loin sur le plateau continental de l'estuaire maritime; 3) que l'étendue des formations de dolomie dans les Appalaches semble très restreinte (Dionne 1999; en préparation). Il en va de même pour la dolomie grise de Beekmantown de la région de Montréal, car jusqu'à maintenant on n'a pas trouvé de trace de cet indicateur dans le secteur du moyen estuaire, entre Québec et l'embouchure du 
Saguenay. Il serait donc surprenant qu'il y ait des cailloux de cette lithologie aux Escoumins.

\section{Les agents de transport et la voie empruntée}

En admettant comme source la plus probable le bassin sédimentaire du Mistassini, le déplacement est de l'ordre de 400 à $500 \mathrm{~km}$. Il s'agit d'un transport de longue distance relativement important (Prest et Nielsen 1987). Dans un premier temps, les cailloux ont indéniablement été déplacés vers le SE par les glaciers. L'écoulement vers la cuvette du Lac-Saint-Jean et le Saguenay, où on trouve aussi des cailloux de dolomie, en particulier des dolos à stromatolites (Dionne 1994), est antérieur à l'écoulement tardif vers le SO observé dans la région immédiate du lac Mistassini et de Chibougamau (Bouchard et Martineau 1985; Prichonnet et al. 1984; Prichonnet et Beaudry 1990; Veillette et Pomares 1991). Contrairement à certains auteurs (Bouchard et al. 1984; Prichonnet et Beaudry 1990), nous croyons que l'écoulement glaciaire vers le SE s'est poursuivi jusqu'à la déglaciation et qu'il a atteint la rive nord du Saint-Laurent estuarien dans la région de Tadoussac/Les Escoumins. En effet, les cailloux de dolomie caractérisent des secteurs côtiers où l'on trouve des diamictons intercalés dans les dépôts marins postglaciaires. Comme beaucoup de cailloux sont dans l'argile et dans les couches de diamicton, ceci implique soit un délestage par des icebergs et/ou des glaces flottantes annuelles, soit un délestage, par une nappe de glace sise à proximité de la rive nord. Les diamictons à l'embouchure du Saguenay, par exemple, contiennent généralement un pourcentage élevé de cailloux de dolomie striés (Dionne 1994).

D'après des relevés récents effectués par Jean Veillette (1999 et communication personnelle, 2000), dans l'arrière pays des Escoumins, les erratiques carbonatés et autres en provenance des formations du bassin sédimentaire de Mistassini et de la région au nord de Chibougamau abondent sur les rives des principaux lacs, en particulier celui du réservoir Pipmuacan situé à environ $160 \mathrm{~km}$ au NO des Escoumins $\left(49^{\circ} 35^{\prime} \mathrm{N}, 70^{\circ} 20^{\prime} 0\right)$.

Une question fondamentale à résoudre est celle de la route (ou des routes) empruntée par la glace à partir d'un dôme situé au NO du lac Mistassini. L'existence de blocs de dolomie, en particulier de dolomie à stromatolites, dans la région du LacSaint-Jean et du. Saguenay, ainsi qu'à son embouchure (Dionne 1986, 1994), implique un courant de glace majeur de 120 à $130 \mathrm{~km}$ de largeur ayant emprunté cet axe en direction du SE (Fig. 6). Compte tenu de la topographie, en particulier du massif du mont Valin, le courant de glace ayant entraîné les cailloux de dolomie du bassin sédimentaire de Mistassini devait avoir une direction générale NNO-SSE $\left(165^{\circ}\right)$ entre le lac Mistassini et la cuvette du Lac-Saint-Jean; de là, l'écoulement glaciaire aurait suivi à peu près l'axe général du Saguenay, orienté plutôt ONO-ESE $\left(115^{\circ}-120^{\circ}\right)$, une direction confirmée par la cartographie des stries dans ce secteur (Dionne 1973). Nous favorisons ce tracé pour le déplacement des erratiques de dolomie et autres lithologies en provenance de la région de Chibougamau - lac Mistassini.

Un deuxième parcours possible serait un écoulement en deux temps, soit d'abord du lac Mistassini vers le réservoir Pipmuacan en passant par le lac Péribonka, sur une distance approximative $320-325 \mathrm{~km}$ selon une direction moyenne NO-
SE à environ $135^{\circ}$; ce courant de glace pourrait avoir eu entre 140 à $150 \mathrm{~km}$ de largeur ou moins; puis dans un deuxième temps, un écoulement NNO-SSE à environ $150^{\circ}$, du réservoir Pipmuacan jusqu'au rivage des Escoumins. Dans ce cas, le courant de glace aurait contourné par le Nord le massif du mont Valin. L'abondance des cailloux de dolomie sur les rives du réservoir Pipmuacan (J.J. Veillette, communication personnelle, 2000) confirme au moins un écoulement glaciaire jusqu'à cet endroit. Il reste à démontrer que, du réservoir Pipmuacan aux Escoumins, les erratiques de dolomie ont été déplacés par un courant de glace de direction SSE. Si c'est le cas, on s'explique mal alors l'absence d'erratiques de dolomie entre Baie-des-Bacons et le cap Colombier, site le plus oriental où nous avons observé une cinquantaine de cailloux de dolomie (Dionne, 2001). Le cap Colombier (48 $49^{\circ} 40^{\prime \prime} \mathrm{N}$, $68^{\circ} 52^{\prime} 45^{\prime \prime} 0$ ) est à environ $135-140 \mathrm{~km}$ au SE du réservoir Pipmuacan et à environ $75 \mathrm{~km}$ au NE de Baie-des-Bacons. Au cap Colombier, les cailloux de dolomie reposent sur un estran argileux (argile de la Mer de Goldthwait) et la direction principale des stries sur le rivage rocheux est NO-SE $\left(130^{\circ}-\right.$ $140^{\circ}$ ), alors que la direction moyenne en ligne droite entre la partie nord du lac Mistassini, le réservoir Pipmuacan et le cap Colombier est de $130^{\circ}$. La concordance nous paraît significative.

Les erratiques de dolomie au cap Colombier semblent donc provenir d'un écoulement glaciaire de direction NO-SE voisin de $130^{\circ}-135^{\circ}$, soit selon un tracé passant au nord du mont Valin. L'absence de cailloux de dolomie entre Baie-desBacons et cap Colombier pourrait être lié à la présence du mont Valin qui aurait dévié l'écoulement des glaces de part et d'autre du massif montagneux.

Quoi qu'il en soit, il convient de mentionner qu'au cap Colombier, nous n'avons pas observé de diamicton dans la terrasse en bordure du rivage; les cailloux proviennent visiblement de l'argile. Il s'agirait donc de cailloux glaciels délestés par les icebergs ou les glaces annuelles durant la submersion de la Mer de Goldthwait. Le petit nombre de cailloux observés est sans doute lié à l'éloignement de la source. Ajoutons, en passant qu'en aval du cap Colombier, nous avons observé seulement quelques petits cailloux (10$20 \mathrm{~cm}$ ) de dolomie sur le rivage de Ragueneau; il n'y en a pas entre ce point et Godbout et, à notre connaissance, personne n'en a signalé plus à l'Est (Dredge 1983).

La grande abondance des cailloux de dolomie ainsi que les quelques cailloux de tillite observés à partir de l'embouchure du Saguenay jusqu'à Baie-des-Bacons, soit sur une cinquantaine de kilomètres, en particulier là où il existe des dépôts meubles en bordure du rivage, témoignent en faveur d'un courant de glace majeur ayant emprunté la dépression du Lac-Saint-Jean et la vallée du Saguenay et peutêtre aussi la vallée attenante de la Sainte-Marguerite. Descendue vers le SE au cours du Wisconsinien, cette masse de glace aurait formé, lors de la déglaciation, une vaste plateforme à l'embouchure du Saguenay. (Dionne et Occhietti 1996) et aurait alors produit une grande quantité d'icebergs qui auraient abandonné leur charge sédimentaire dans le secteur de Grandes-Bergeronnes - Les Escoumins au début de la submersion post-glaciaire, ce qui expliquerait la présence de lits et de poches de diamicton dans les rythmites limonoargileuses des terrasses côtières. Il est vraisemblable aussi que 


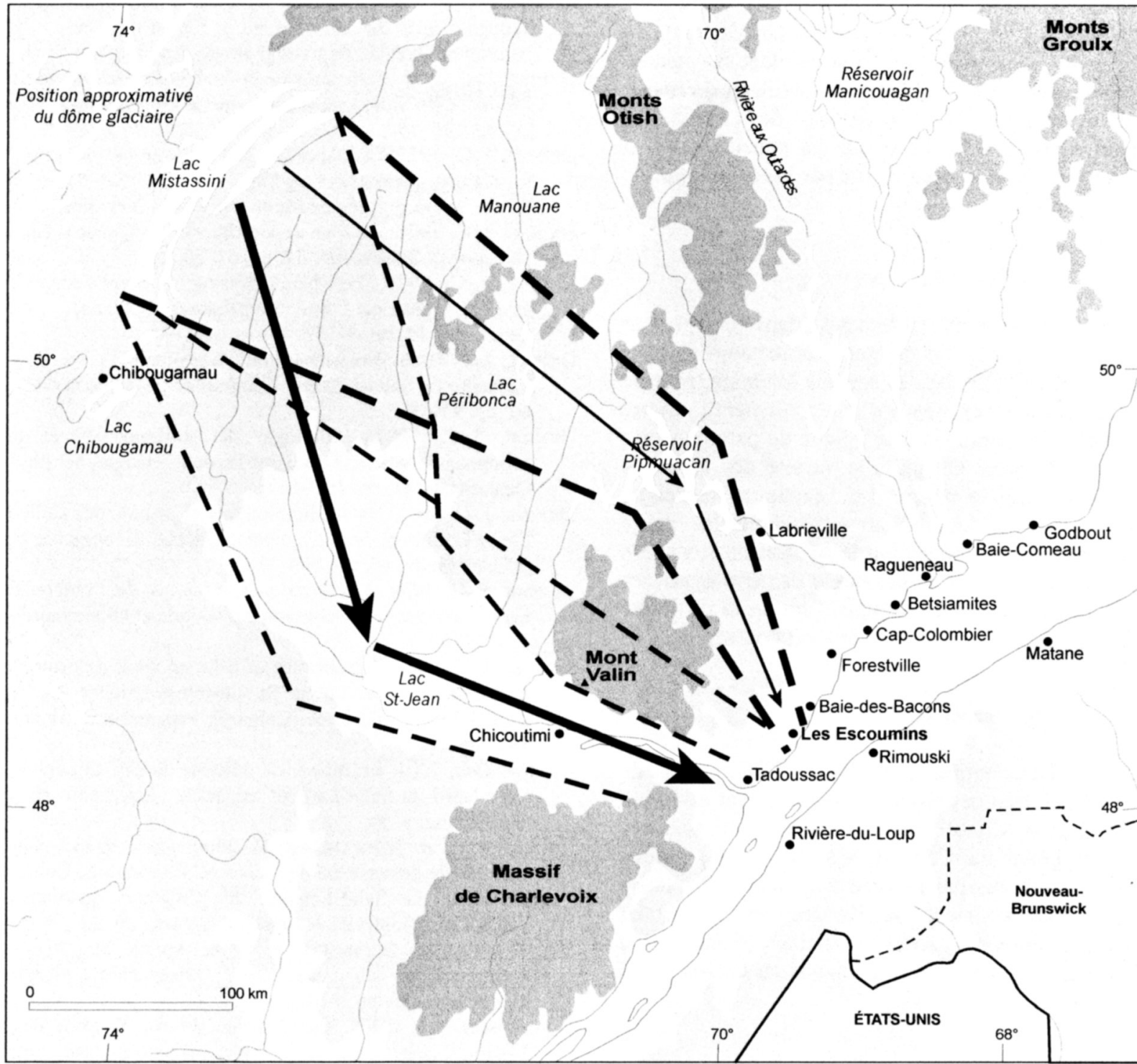

Altitude (mètres)
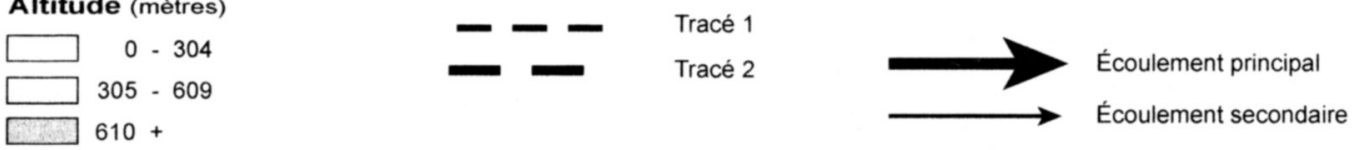

Fig. 6. Tracés possibles de l'écoulement des glaces entre le lac Mistassini et Les Escoumins.

la langue de glace qui a occupé tardivement le Saguenay ait contenu une certaine quantité de cailloux erratiques provenant de la région du lac Mistassini, d'où une dispersion tardive dans la Mer de Goldthwait.

Dans la région des Escoumins, le dernier écoulement glaciaire est vers le $\mathrm{NE}$ à $60^{\circ}$, ce qui implique la présence d'une glace active dans la vallée du Saint-Laurent au cours de la déglaciation. La dispersion des erratiques en provenance du bassin sédimentaire de Mistassini serait donc due à la fois à un courant glaciaire dirigé d'abord vers la cuvette du Lac-SaintJean, puis ensuite vers le Saguenay, et à un délestage par des icebergs issus de cette masse de glace wisconsinienne qui a occupé le secteur aval du Saguenay jusque vers 10-10,5 ka (Dionne et Occhietti 1996).
Les cailloux de dolomie du rivage entre Les Escoumins et Baie-des-Bacons se rencontrent dans un secteur caractérisé par un large estran argileux correspondant à une surface d'érosion qui aboutit à une falaise encore active entaillant des dépôts meubles mis en place lors de la déglaciation et l'épisode de la Mer de Goldthwait; ces dépôts contiennent souvent des lits de diamicton d'épaisseur variable; certains contiennent des cailloux de dolomie, d'autres pas, alors qu'on en trouve dans l'argile; il y a donc eu des délestages contemporains de la mise en place des dépôts, ce qui suggère une activité relativement importante des icebergs. De nos jours, les cailloux du rivage dans la région des Escoumins subissent certains déplacements locaux par les glaces flottantes. Toutefois, les méga-blocs sont peu mobiles; il en est de même des blocs constituant des 
dallages à la surface de la batture argileuse; les cailloux les plus mobiles sont les petits et les moyens $(25-50 \mathrm{~cm})$, en particulier ceux du rivage supérieur ou de la plage au pied de la falaise. Quoi qu'il en soit, la majorité des cailloux du rivage des Escoumins proviennent de l'érosion des formations meubles quaternaires en bordure et ont été peu déplacés. Il s'agit donc du résidu grossier (lag) laissé par l'érosion littorale au cours de l'Holocène.

\section{CONCLUSION}

La présence d'éléments carbonatés dans un secteur composé essentiellement de roches ignées et métamorphiques a permis de mettre en évidence des apports lointains (plus de $400 \mathrm{~km}$ ) èt de confirmer l'existence d'un écoulement glaciaire vers le SE à partir d'un dôme et d'une ligne de partage situés au NO du lac Mistassini. De plus, la variété des types de dolomie rencontrées sur le rivage des Escoumins renseigne utilement sur la nature des formations carbonatées d'âge protérozoïque du bassin sédimentaire de Mistassini. Il s'avère donc avantageux de connaître la lithologie des erratiques des rives du Saint-Laurent estuarien compte tenu de la difficulté d'effectuer des relevés exhaustifs dans la forêt boréale.

\section{REMERCIEMENTS}

La présente contribution fait partie d'un projet de recherche sur l'Évolution des rives du Saint-Laurent estuarien subventionné par le Conseil de recherches en sciences naturelles et en génie du Canada (CRSNG). Les figures dessinées ont été réalisées par Andrée Gauthier, Laboratoire de cartographie, Département de Géographie, Université Laval. Nous remercions Jean Veillette (Commission géologique du Canada) avec qui nous avons eu de fructueux échanges.

\section{RÉFÉRENCES}

Avramtchev, L. 1985. Carte géologique du Québec. Ministère de l'Énergie et des Ressources, Québec, carte no 2000, DV-84-02.

BARAGAR, W.R.A. 1967. Wukuach Lake map-area, QuébecLabrador. Geological Survey of Canada, Memoir 344, 174 p.

Bouchard, M.A., CADIEUX, B. \& Goutier, F. 1984. L'origine et les caractéristiques des lithofaciès du till dans le secteur nord du lac Albanel, Québec: une étude de la dispersion clastique. Canadian Institute of Mining and Metallurgy, Special Volume 34, pp. 244-260.

Bouchard, M.A. \& MartineaU, G. 1985. Southeastward ice flow in central Québec and its paleogeography significance. Canadian Journal of Earth Sciences, 22, pp. 1536-1541.

Boulton, G.S., SMITh, D.G., JONES, A.S. \& NewsOME, J. 1985. Glacial geology and glaciology of the last mid-latitude ice sheets. Journal of the Geological Society of London, 142, pp. 447-474.

CATY, J.L. 1976. Région du lac Mistassini: stratigraphie et sédimentologie de la formation de Papaskwasati. Ministère de l'Énergie et des Ressources, Québec, Rapport géologique 423, $270 \mathrm{p}$.

Clark, T.H. 1972. Région de Montréal, Ministère des Richesses naturelles, Québec, Rapport géologique 152, 244 p.
DimRoth, E. 1978. Région de la fosse du Labrador - Labrador Trough area: $50^{\circ} 30^{\prime}-56^{\circ} 30^{\prime}$. Ministère des Richesses naturelles, Québec, Rapport géologique 193, 396 p.

DiONNE, J.-C. 1972. Caractéristiques des blocs erratiques des rives de l'estuaire du Saint-Laurent. Revue de Géographie de Montréal, 26, pp. 125-152.

DiONNE, J.-C. 1973. La dispersion des cailloux ordoviciens dans les formations quaternaires au Saguenay / Lac-Saint-Jean, Québec. Revue de Géographie de Montréal, 27, pp. 339-364.

DionNE, J.-C. 1977. La Mer de Goldthwait au Québec. Géographie physique et Quaternaire, 31, pp. 61-80.

DiONNE, J.-C. 1979. Les blocs d'estran à Saint-Fabien-sur-Mer, estuaire maritime du Saint-Laurent, Québec. Maritime Sediments, 15, pp. 5-13.

DiONNE, J.-C. 1986. Blocs de dolomie à stromatolites sur les rives de l'estuaire du Saint-Laurent. Géographie physique et Quaternaire, 40, pp. 93-98.

DionNE, J.-C. 1987. Lithologie des cailloux de la baie de Montmagny, côte sud du Saint-Laurent. Géographie physique et Quaternaire, 41, pp. 161-169.

DiONNE, J.-C. 1991. Nature lithologique et origine des cailloux dans l'anse Hamilton, Saint-Romuald, Québec. Géographie physique et Quaternaire, 45, pp. 219-230.

DiONNE, J.-C. 1994. Les erratiques lointains de l'embouchure du Saguenay, Québec. Géographie physique et Quaternaire, 48, pp. 179-194.

DiONNE, J.-C. 1999. Significance of Silurian coral dolostone erratics in the shore zone, Lower St. Lawrence estuary. CANQUA CGRG Joint Conference (Calgary), Program and Abstracts, pp. 18-19.

DIONNE, J.-C. 2001. Erratiques de dolomie au cap Colombier, haute Côte Nord du Saint-Laurent estuarien. Géographie physique et Quaternaire. v. 55.

Dionne, J.-C. \& BernatCheZ, P. 2000. Un erratique peu banal: cailloux de dolomie sur le rivage des Escoumins, Côte Nord de l'estuaire du Saint-Laurent. $\mathbf{8}^{\mathrm{e}}$ Colloque quadriannuel de l'AQQUA (Montréal), Résumés-Abstracts, pp. 27-28.

DIONNE, J.-C. \& OCCHIETTI, S. 1996. Aperçu du Quaternaire à l'embouchure du Saguenay. Géographie physique et Quaternaire, 50, pp. 5-34.

DonALDSON, J.A. 1963. Stromatolites in the Denault Formation, Marion Lake, coast of Labrador, Newfoundland. Geological Survey of Canada, Bulletin 102, 33 p.

DONALDSON, J.A. 1966. Marion Lake map-area, QuébecNewfoundland. Geological Survey of Canada, Memoir 338, $85 \mathrm{p}$.

DREDGE, L.A. 1983. Surficial geology of the Sept-Iles area, Québec North Shore. Geological Survey of Canada, Memoir 408: 40 p.

DRESSER, B. 1979. Région de la fosse du Labrador $\left(56^{\circ} 30^{\prime}-57^{\circ} 15^{\prime}\right)$. Ministère des Richesses naturelles, Québec, Rapport géologique $195,117 \mathrm{p}$

DYKE, A.S. \& PREST, V.K. 1987a. Paleogeography of northern North America (18-5 ka). Geological Survey of Canada, Map 1703A, scale $1 / 12500000$.

DYKe, A.S. \& PREST, V.K. 1987b. Late Wisconsinan and Holocene retreat of the Laurentide Ice sheet. Geological Survey of Canada, Map 1702A, scale 1/5000 000.

FisheR, D.A., ReEh, N. \& LANGley, K. 1985. Objective reconstruction of the Late Wisconsinan Laurentide Ice Sheet and the significance of deformable beds. Géographie physique et Quaternaire, 39, pp. 229-238.

Franconi, S., Sharma, K.N.M. \& Laurin, A.F. 1975. Région des rivières Betsiamites et Moisie (Grenville 1968-1969). Ministère des richesses naturelles, Québec, Rapport géologique 162, $149 \mathrm{p}$. 
GreiG, E.W. 1952. La région de Pontgravé-Bergeronnes, comté de Saguenay. Ministère des Mines, Québec, Rapport géologique $32,32 \mathrm{p}$.

Gullbault, P. 2000. Microfaune de six sédiments Quaternaires marins de la région du Bas-du-Fleuve. BRAQ-Stratigraphie, Montréal, Rapport non publié, $13 \mathrm{p}$.

HofmanN, H.J. 1969. Attributes of stromatolites. Geological Survey of Canada, Paper 69-39, $58 \mathrm{p}$.

HOFMANN, H.J. 1973. Stromatolites: characteristics and utility. EarthScience Reviews, 9, pp. 339-373.

LaURIN, A.F. \& Sharma, K.N.M. 1975. Région des rivières Mistassini - Péribonca - Saguenay (Grenville, 1965-1967). Ministère des Richesses naturelles, Québec, Rapport géologique $161,89 \mathrm{p}$.

NEILSON, J.M. 1953. Albanel Lake area, Mistassini territory. Ministère des Mines, Québec, Rapport géologique 53, $35 \mathrm{p}$.

Occhietti, S., Parent, M., Shilts, W.W., Dionne, J.C., Govare, E. \& HARMAND, D. 2001. Late Wisconsinan glacial dynamics, deglaciation and marine invasion in southern Québec. In Deglacial history and relative sea level changes, northern New England and adjacent Canada. Edited by T.K. Weddle and M.J. Retelle, Special Paper 351, pp. 245-272.

Parent, M., Paradis, S.J. \& BoISVerT, E. 1995. Ice-flow patterns and glacial transport in the eastern Hudson Bay region: implication for the Late Quaternary dynamics of the Laurentide Ice Sheet. Canadian Journal of Earth Sciences, 32, pp. 20572070.

PREST, V.K. \& NielSEN, E. 1987. The Laurentide ice Sheet and longdistance transport. Geological Survey of Finland, Special Paper 3, pp. 91-101.

PrichonNet, G. \& BEaUdRy, L.M. 1990. Évidence d'un écoulement glaciaire sud, antérieur à l'écoulement sud-ouest du Wisconsinien supérieur, région de Chapais, Québec. In Recherches en cours, Partie C, Commission géologique du Canada, Étude 90-1C, pp. 331-338.
Prichonnet, G., Martineau, G. \& Brisson, L. 1984. Les dépôts quaternaires de la région de Chibougamau, Québec. Géographie physique et Quaternaire, 38, pp. 287-304.

RONDOT, J. 1986. Géologie de la région de Forestville - Les Escoumins. Ministère de l'Énergie et des Ressources, Québec. Rapport géologique ET 85-05, 47 p.

SlivitsKY, A., St-JUlien, P. \& LaChambre, G. 1991. Synthèse géologique du Cambro-Ordovicien de la Gaspésie. Ministère de l'Énergie et des Ressources, Québec, ET 88-14, 61 p.

Tremblay, P. \& Bourque, P.A. (éds.) 1991. Carte géotouristique du Bas-Saint-Laurent et de la Gaspésie. Ministère de l'Énergie et des Ressources, Québec, carte GT 91-03, échelle 1/500,000.

TWENHOFEL, W.H. 1938. Geology and paleontology of the Mingan Islands, Québec. Geological Society of America, Special Paper $11,132 \mathrm{p}$.

VEILLETTE, J.J. 1986. Former southwesterly ice flows in the AbitibiTemiscamingue region: implication for the configuration of the Late Wisconsinan ice sheet. Canadian Journal of Earth Sciences, 23, pp. 1724-1741.

VEILLETTE, J.J. 1999. Les erratiques protérozoïques du lac Mistassini: un traceur exceptionnel. Bulletin de l'AQQUA, 25 (2), pp. 22.

VEILLETTE, J.J., DYKE, A.S. \& ROY, M. 1999. Ice-flow evolution of the Labrador sector of the Laurentide Ice Sheet: a review with new evidence from northern Québec. Quaternary Science Reviews, 18, pp. 993-1019.

VeIllette, J.J. \& POMARES, J.S. 1991. Older ice flows in the Matagami-Chapais area, Québec. In Current Research, Part C, Geological Survey of Canada, Paper 91-1C, pp. 43-48.

WADDINGTON, G.W. 1950. Les dépôts de calcaire de la région de Mingan, comté de Saguenay. Ministère des Mines, Québec, Rapport géologique 42, Partie 2, 13 p.

WAHL, W.G. 1953. Temiscamingue River area. Département des Mines, Québec, Rapport géologique 54, 32 p.

Editorial responsibility: Graham L. Williams 